\title{
Insuficiência Cardíaca de Alto Débito Devida a Fístula Coronária
}

\author{
O rlando Petrucci Jr, Pedro Paulo M. de O liveira, Cid de Abreu Leme Jr, 0 távio Rizzi Coelho, Fernando \\ A. Barca Schellini, Eduardo Arantes Nogueira, Reinaldo W. Vieira, Domingo Marcolino Braile
}

Campinas, SP

Em mulher de 45 anos, apresentando quadro de insuficiência cardíaca classe funcional III e dor torácica atípica, foi evidenciada dilatação importante da coronária direita e sua drenagem para o átrio direito, demonstrada na ecocardiografia transtorácica e confirmada pela angiografia coronária. A paciente foi submetida a cirurgia, encontrando-se um ramo único desembocando no átrio direito, que foi ligado. Sua evolução pós-operatória foi boa estando, há três anos, livre de qualquer sintomatologia.

\section{High Output Heart Failure due to a Coro- nary Fistula}

A 45 year-old woman complaining of heart failure symptoms (New York Heart Association -class III) and a non typical thoracic pain was submitted to a transthoracic echocardiogram which showed a very dilated coronary artery and a fistula to the right atrium. The angiograms confirmed the same findings. She underwent open heart surgery which confirmed the diagnosis. Fistula ligation was then undertaken. She remains symptom-free three years after the operation.
Fístulas arteriovenosas coronária são consideradas achado raro, mas que são diagnosticadas prontamente.

A sua incidência é estimada em 0,1 a 0,2 \% em angiografia coronária ${ }^{1}$, normalmente estas fístulas são anomalias isoladas, mas podem estar associadas a doenças congênitas ou adquiridas, associada a arteriosclerose severa ou doença sistêmica. Podem ser causadas por trauma fechado, por ferimentos de arma branca ou após biópsias endomiocárdicas repetidas em pacientes com transplante cardíaco.

Há relatos também de fístulas coronárias associadas a eletrodos permanentes de marcapasso por erosão, após cirurgia cardíaca, angioplastia coronária ou infarto agudo do miocárdio

Neste relato descrevemos uma fístula de coronária direita para o átrio direito (AD), causando insuficiência cardíaca de alto débito.

Universidade Estadual de Campinas - UNICAMP

Correspondência: Orlando Petrucci Jr - Rua Carlos Guimarães, 248/92 - 13024200 - Campinas, SP

Recebido para publicação em 9/9/97

Aceito em 16/10/97

\section{Relato do Caso}

Mulher de 45 anos, do lar, procurou nosso serviço com queixa de cansaço e dispnéia aos pequenos esforços, ocasionalmente, acompanhados de precordialgia atípica; cujos sintomas haviam iniciado há, aproximadamente, um mês.

Ao exame físico apresentava-se em regular estado geral, taquidispnéica, acianótica, afebril e discreto edema de

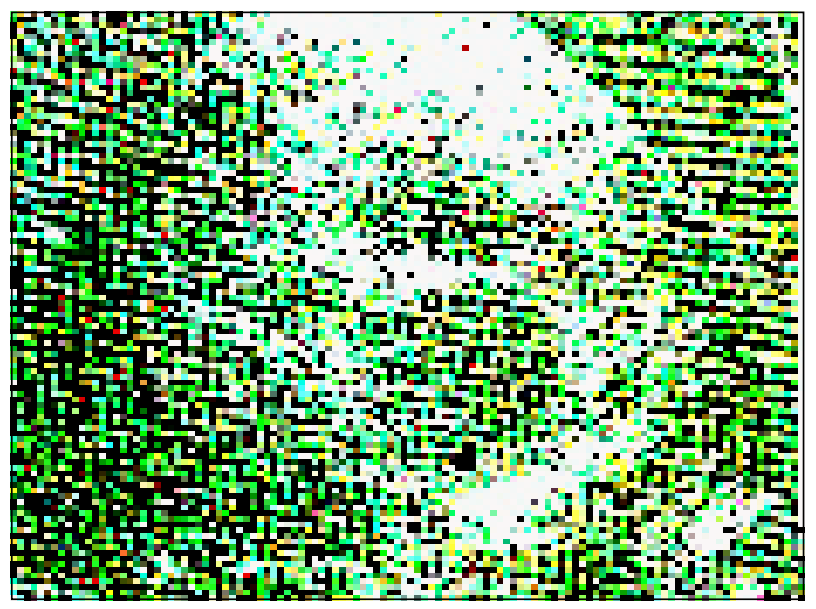

Fig. 1 - Ecografia transtorácica demonstrando coronária direita dilatada desde o seu início 


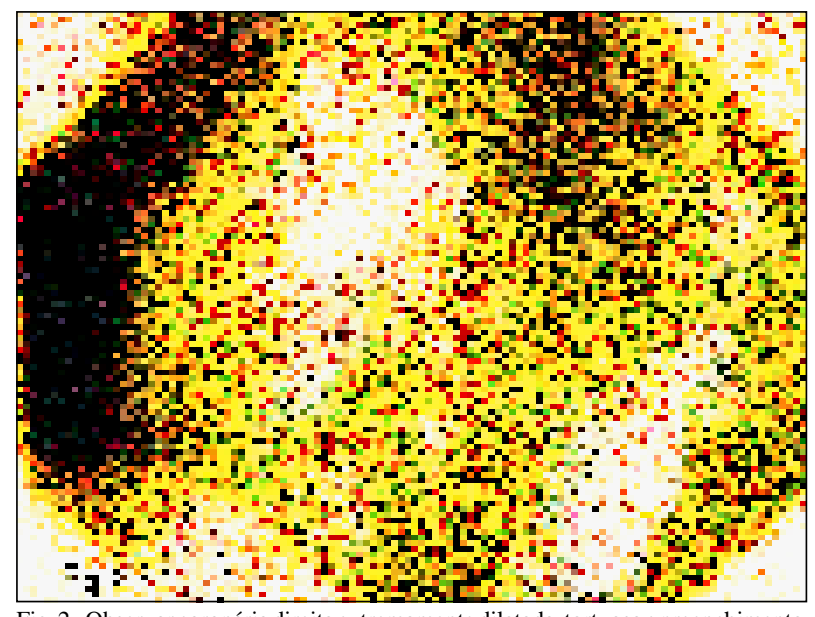

Fig. 2 - Observar coronária direita extremamente dilatada, tortuosa e preenchimento do átrio direito pelo contraste.

membros inferiores, pulsos regulares e simétricos. À ausculta cardíaca apresentava sopro sistodiastólico em região paraesternal direita rude, freqüência cardíaca de $120 \mathrm{bpm}$ e pressão arterial de 140/80mmHg.

O eletrocardiograma (ECG) revelou ritmo sinusal sem alteração de repolarização e a radiografia de tórax, área cardíaca dentro da normalidade.

Realizado ecocardiografia transtorácica que evidenciou artéria coronária direita dilatada com aproximadamente $11 \mathrm{~mm}$ (fig. 1). Seguiu-se a realização de angiografia coronária que evidenciando coronária direita dilatada e com trajeto fistuloso para o $\mathrm{AD}$, não se evidenciando quaisquer outras alterações (fig. 2).

A paciente foi submetida à cirurgia, realizando-se esternotomia mediana e, após se ter estabelecido a circulação extracorpórea convencional, o $\mathrm{AD}$ foi aberto e visibilizou-se um ramo da coronária direita desembocando na parede posterior do $\mathrm{AD}$ na junção cavoatrial superior. Foi realizada ligadura do ramo arterial anômalo. A paciente apresentou pós-operatório sem intercorrências, não apresentando qualquer queixa cardiovascular no seguimento de três anos (fig. 3).

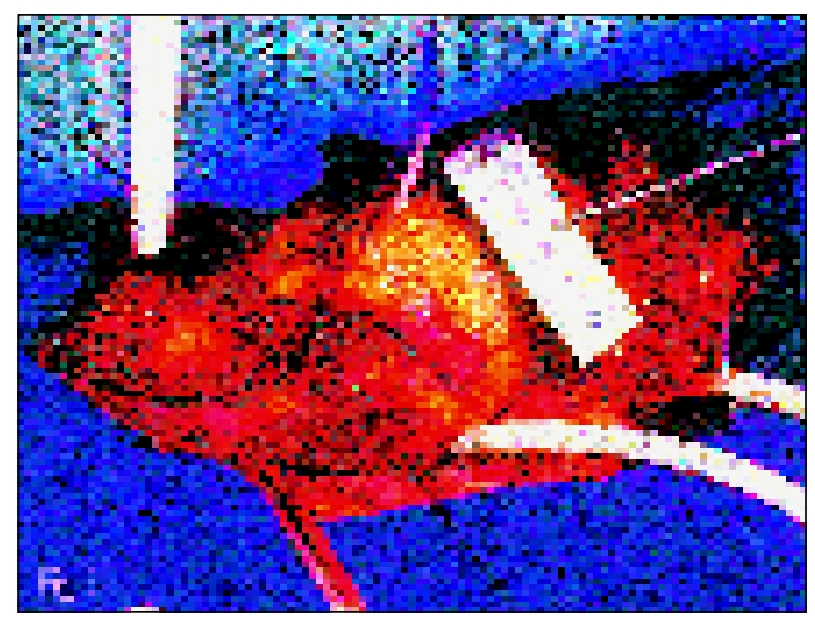

Fig. 3 - Achado intra-operatório com coronária direita extremamente dilatada.

\section{Discussão}

Fístulas arteriovenosas coronárias em angiografias não selecionadas na população de adultos para avaliação de dor torácica são raras e apresentam normalmente evolução benigna ${ }^{2}$.

A grande maioria das fístulas coronárias é congênita e não está associada a outras anomalias cardíacas. Em sua grande parte são originadas da coronária direita que, na maioria das vezes, comunicam-se com as câmaras direitas. Em geral, não apresentam distúrbios hemodinâmicos relacionados a anomalia e o shunt esquerda direita é pouco significativo, mas podem ocasionalmente apresentar isquemia crônica ou aguda do miocárdio, insuficiência cardíaca, endocardite, infecções recorrentes do trato respiratório ou, em alguns casos raros, hipertensão pulmonar.

No presente relato, a paciente apresentava-se em insuficiência cardíaca franca por alto débito cardíaco, que se resolveu após a correção cirúrgica.

As artérias coronárias se desenvolvem cedo na embriogênese, entre a $6^{\mathrm{a}}$ e $8^{\mathrm{a}}$ semanas. A $1^{\mathrm{a}}$ estrutura das artérias coronárias consiste de uma rede capilar miocárdica, que é formada de células endoteliais. O sistema arterial coronário forma-se ao redor do tronco arterioso, e a sua estrutura principal começa a se desenvolver mais na porção que irá dar a futura aorta, quando de sua bipartição unindo-se à rede capilar miocárdica. As fístulas coronárias podem se desenvolver quando há um aumento da rede capilar durante a embriogênese da circulação coronária ou quando a artéria coronária principal permanece aderida ao tronco da pulmonar.

O diagnóstico clínico deve ser suspeitado com a ausculta de sopro contínuo em uma localização não usual para canal arterial.

O diagnóstico conclusivo realiza-se pela angiografia coronária. Outros métodos diagnósticos têm sido propostos na literatura, como: ecocardiografia transtorácica e/ou transesofágica ou ressonância magnética nuclear ${ }^{3}$.

No presente caso, a ecocardiografia transtorácica já evidenciou dilatação importante da coronária direita, o que levou à suspeita da fístula coronária.

O papel suplementar da ecocardiografia transesofágica junto à angiografia coronária para o diagnóstico de anomalias das artérias coronárias tem sido estabelecido em uma série selecionada de Giannoccaro e $\mathrm{col}^{4}$, onde a ecocardiografia transesofágica não só confirmou a presença de anomalias detectadas pela angiografia como auxiliou na localização da drenagem de fístulas coronárias.

Apesar das fistulas coronárias apresentarem uma evolução benigna, de modo geral, o seu fechamento espontâneo é muito raro. Tem sido postulado que as fístulas coronárias pequenas têm história relativamente benigna .

Quando se diagnostica a fístula em adultos, o paciente deve ser avaliado para se ter o diagnóstico diferencial entre a etiologia congênita ou adquirida pois, clinicamente as duas condições podem ser idênticas. A diferenciação pode ser feita pela história, pelo aspecto da coronária na angiografia, observando-se o calibre da artéria comprometi- 
da. A artéria comprometida, está usualmente de calibre normal na doença aterosclerótica, mas é dilatada e tortuosa nas fístulas congênitas.

No presente relato, a paciente apresentava a artéria coronária direita dilatada e bastante tortuosa no seu terço proximal, muito característico de origem congênita.

A correção pode ser por métodos não invasivos como técnicas de embolização de partículas de Ivalon, coils e balões, ou cirúrgicos mesmo em pacientes idosos com grande sucesso.

Fístulas coronárias congênitas apresentam um amplo espectro clínico, ocorrendo sintomatologia no jovem, assim como no idoso. A razão da apresentação clínica deste caso deve-se a magnitude do shunt esquerda-direita, na ausência de outras alterações patológicas associadas ou doença aterosclerótica coronária importante.

Quando o diagnóstico é feito em pacientes assintomáticos, uma avaliação periódica com exames não invasivos deve ser realizada. Na presença de alterações, como isquêmicas no ECG, teste de esforço positivo ou distúrbios de ritmo, a fístula deve ser fechada, sendo que a escolha quanto ao procedimento cirúrgico ou não cirúrgico, dependerá do tipo, local, tamanho e necessidade de outros procedimentos associados. Acreditamos que a profilaxia para endocardite deve ser realizada sempre nesta doença ${ }^{5}$.

\section{Referências}

1. Gillebert C, Van Hoof R, Van de WerfF, Piessens, De Geest H- Coronary artery fistulas in an adult population. Eur Heart J 1986; 7: 437-43.

2. Vavuranakis M, Bush C, Boudoulas H - Coronary artery fistulas in adults: incidence, angiographic characteristics, natural history. Cathet Cardiovasc Diagnosis 1995; 35: 116-20.

3. Kubota H, Suzuki T, Murata K - Cine magnetic resonance imaging for diagno- sis of right coronary arterial-ventricular fistula. Chest 1991; 100: 735-7.

4. Giannoccaro PJ, Sochowski RA, Morton BC, Chan KL-Complementary role of transesophageal echocardiography to coronary angiography in the assessment of coronary artery anomalies. Br Heart J 1993; 70: 70-4.

5. Alkhulaifi AM, Horner SM, Pugsley WB, Swanton RH-Coronary artery fistulas presenting with bacterial endocarditis. Ann Thorac Surg 1995; 60: 202-4. 